\title{
Sexual Minority Mental Health
}

Brooke G. Rogers, Ph.D., M.P.H., ${ }^{1}$ Audrey Harkness, Ph.D., ${ }^{2}$ and Craig Rodriguez-Seijas, Ph.D. ${ }^{3}$

1. Department of Medicine, Warren Alpert Medical School of Brown University, 11 Fourth Street, Providence, RI, 02903 USA

2. Department of Public Health Sciences, University of Miami Miller School of Medicine, 1600 NW 10th Ave \#1140, Miami, FL 33136, USA

3. Department of Psychology, University of Michigan, 530 Church Street, Ann Arbor, MI 48109, USA

\section{Author Note}

Correspondence concerning this article should be addressed to corresponding author Brooke G. Rogers and 11 Fourth Street Providence, RI 02906 and brooke_rogers@brown.edu. 


\begin{abstract}
Sexual minority clients are more likely to experience emotional disorders and other psychiatric conditions than the general population. This health disparity is not attributable to an individual's sexual minority identity, but rather, the social milieu that produces a unique set of stigma-related stressors. Therefore, mental health disparities affecting sexual minority communities are best understood through a minority stress framework. This chapter outlines mental health disparities affecting sexual minority communities, tailored evidence-based treatments, and clinical considerations for working with sexual minority clients. We hope this chapter can offer pragmatic guidance on how best to tailor intervention approaches given the unique needs and lived experiences of this population.
\end{abstract}

Keywords: sexual minority; lesbian; gay; bisexual; mental health; disparities; substance use; trauma; disordered eating; body image; transdiagnostic; affirmative therapy 


\subsection{Introduction}

This chapter is focused on clinical considerations for working with individuals whose sexual orientation identities fall within the umbrella of "sexual minority," including individuals who identify as gay, lesbian, bisexual, pansexual, and other queer or non-heterosexual identities. In writing this chapter, we acknowledge that the focus is on sexual minority clients, which in some cases, also includes individuals who identify as transgender, gender non-binary, and/or gender diverse. Interventions focused on addressing the specific needs and experiences of gender diverse clients are critically needed and important as well, and not the explicit focus of the current chapter. However, many of the cross-cutting principles germane to providing affirmative therapy may also be appropriate to consider when working with gender diverse clients. Finally, we note that gender is not a binary construct; yet much of the existing literature on sexual minority persons has focused on sexual minority men or sexual minority women and has not addressed the gender continuum. This is an area for improvement within the field. In the absence of this literature we attempt to contextualize our perspectives on working with sexual minority clients within the existing literature.

It is well documented that sexual minority persons experience higher prevalence of mental health disorders than their heterosexual peers (Brim et al., 1999; Cochran, Mays and Sullivan, 2003; Pakula et al., 2016; Cochran, Björkenstam and Mays, 2017; Bränström and Pachankis, 2018). Estimates range depending on the study, with prevalence for some disorders as high as five times that of their heterosexual counterparts (Cochran, Mays and Sullivan, 2003). This is true across a variety of psychopathology including depression, anxiety, trauma, interpersonal violence, and substance use. These disparities are best understood as resulting from a myriad of social forces that both potentiate baseline stress and predispose sexual minority individuals to experiencing a host of negative psychosocial events, including discrimination, stigma, and other forms of minority stress (Meyer, 2003).

Congruent with higher prevalence of psychosocial concerns, individuals who identify as sexual minorities also seek mental health services at higher rates than the general population (Cochran, Mays and Sullivan, 2003; Cochran, Björkenstam and Mays, 2017). Sexual minority men reported seeing a mental health provider in the past year more than twice as often as heterosexual men (19.4\% vs. $7.5 \%$ ) and sexual minority women reported seeing a mental health provider almost three times as often as heterosexual women (33.0\% vs. $10.7 \%)$ in the past year (Cochran, Mays and Sullivan, 2003). Still, there is a paucity of evidence-based treatments that are readily accessible for community clinicians to utilize to provide culturally congruent and affirming counseling services to sexual minority clients. As a result, it is unclear the extent to which sexual minorities receiving mental health services receive care that is evidence-based and culturally responsive to the needs of lesbian, gay, bisexual, transgender, and queer persons (LGBTQ+).

To address this gap, there has been an emergence of researchers and clinicians who are focused on developing and testing evidence-based treatments that have been specifically adapted for sexual minority clients (Craig, Austin and Alessi, 2013; Craig and Austin, 2016; Blashill et al., 2017; Brown et al., 2017; Craig et al., 2019; Pachankis et al., 2019, 2020). 
Broadly, "LGBTQ-affirmative" describes a therapeutic approach that positively embraces individuals with an LGBTQ+ identity and acknowledges and addresses negative experiences due to heterosexism, homophobia, and transphobia and their impact on mental health. LGBTQaffirmative approaches also generally leverage the resilience and strengths of the LGBTQ community, rather than being limited to a deficit-reduction model. As efficacy and effectiveness data are gathered, it will be important that these interventions are disseminated readily into community treatment settings to support their use in everyday practice.

There have also been efforts to identify the "key principles" of LGBTQ-affirmative psychotherapy practice (American Psychological Association, 2012; Pachankis, 2014, 2018), including normalizing LGBTQ+ identities, acknowledging explicit and implicit biases, understanding specific challenges for subgroups within the LGBTQ+ community, increasing knowledge of relevant community issues through ongoing education, consultation, and supervision, and presenting research findings in a way that is balanced and mindful of how they could be misused, among others. These principles will be explicated and reviewed in greater detail later in the chapter. In the absence of specific interventions for a specific presenting concern, these guidelines could be integrated with existing evidence-based treatments. Within this chapter, we review the state of the science with regards to evidence-based sexual minority affirming mental health interventions, treatment adaptations and applications, and directions for the future of this field.

\subsection{Mental Health Disparities}

Sexual minority individuals experience higher prevalence of mental health conditions than their heterosexual peers. For instance, Cochran and colleagues (2003) examined data from the 1995-1996 McArthur Foundation Study of Mid-Life (Brim et al., 1999) to assess differences in rates of mental health concern by sexual orientation (Cochran, Mays and Sullivan, 2003). The original study was unique in that it included a self-reported sexual identity measure, which typically had been excluded from prior large, population-based studies. It included a random sample of individuals ages 25-75 years who were non-institutionalized, English speaking adults. Cochran and colleagues (2003) analyzed the McArthur Foundation data and found that sexual minority men were three times more likely to meet criteria for depression and 4.7 times more likely to meet criteria for panic disorder than heterosexual men. Nearly half (49\%) of sexual minority men meeting criteria for a mental health disorder had two or more disorders, in comparison to only $30 \%$ of heterosexual men meeting criteria for two or more disorders. Sexual minority women were also more likely to be diagnosed with more than two disorders than heterosexual women. Approximately $54 \%$ of those sexual minority women meeting criteria for any disorder met for two or more disorders compared to only $30 \%$ of heterosexual women (Cochran, Mays and Sullivan, 2003). This finding of LGBTQ persons having higher prevalence of mental health disorders has been replicated internationally. For example, Pakula and colleagues examined data from the Canadian Community Health Survey and found that lesbian, gay, and bisexual (LGB) persons had higher rates of anxiety, depressive, and alcohol use disorders compared to heterosexual persons (Pakula et al., 2016). Additionally, Bränström and colleagues used the Swedish National Public Health Survey to examine sexual minority related 
health disparities and found significant mental health and substance use disparities based on sexual orientation (Bränström and Pachankis, 2018).

Sexual minority individuals are more likely to experience trauma as a result of minority stress and discriminatory experiences, including peer victimization and bullying, parental emotional and physical abuse, and sexual abuse (Friedman et al., 2011). Social isolation and peer rejection may explain the disproportionate prevalence of interpersonal trauma experiences among sexual minority persons. Traumas experienced by people who identify as sexual minorities are multifaceted and have a lasting impact on mental health and substance use (O'Cleirigh, Safren and Mayer, 2012). Although structural interventions that seek to prevent victimization from occurring in the first place are needed, most research has focused on creating trauma interventions to address specific traumas and their sequelae for sexual minority clients, at an individual level. For example, work by O'Cleirigh and colleagues addresses childhood sexual abuse and sexual compulsivity among sexual minority men (Taylor et al., 2018).

Body image and eating pathology are also more common among sexual minority individuals than their heterosexual peers (Austin et al., 2012; Calzo et al., 2017). Higher prevalence of body dissatisfaction and attempts to control weight through drastic measures including dieting, exercise routines, and supplements are noted as early as adolescence, with sexual minority populations having higher levels of disordered eating and weight control behavior than their heterosexual counterparts (Watson et al., 2017). Additionally, eating disorders meeting diagnostic criteria are at least as common, and in some studies more common, among sexual minority women (Meneguzzo et al., 2018) and significantly more common among sexual minority men compared to their heterosexual peers (Feldman and Meyer, 2007). For example, in one clinical sample of individuals with eating disorders, $42 \%$ of men in the sample identified as sexual minorities (even though in the general population, only $3 \%$ of men identify as sexual minorities) (Carlat, Camargo and Herzog, 1997). Sexual minority women compared to heterosexual women report more bingeing and purging, lower body satisfaction, and higher drive for thinness (Calzo et al., 2017). Many interventions have been designed for younger adult women without attention to sexual orientation; as a result, few trials have collected data on how many of the women in these trials identified as sexual minorities. However, based on population data and assuming these were representative samples, sexual minority women likely comprised a portion of these larger trials. Unfortunately, as is a common limitation in the broader literature of evidence-based interventions (Pachankis, 2018), we were unable to find moderator analyses to compare their effectiveness for heterosexual and sexual minority women. Sexual minority men also experience more body dissatisfaction, have a greater discrepancy between actual and ideal body size and shape, engage in more disordered eating behaviors, and are more likely to meet criteria for an eating disorder and experience body image disturbance than their heterosexual counterparts (Blashill, 2010; Matthews-Ewald, Zullig and Ward, 2014). This has prompted the development of adapted interventions for this population, which have shown efficacy for disordered eating behavior and body image disturbance and are further discussed later (Brown and Keel, 2015; Blashill et al., 2017; Brown et al., 2017).

Substance use disorders are also diagnosed more frequently among sexual minority persons. According to the 2018 National Survey on Drug Use and Health, a comprehensive survey that 
assesses drug use, mental health symptoms, and treatment utilization, sexual minority persons use substances at higher prevalence than the general population (Substance Abuse and Mental Health Services Administration and U.S. Department of Health and Human Services, 2018). All 50 states and D.C. are represented in the survey, which assesses approximately 67,500 households and is intended to be nationally representative. The study examined substance use and mental health among sexual minority persons and found $16.5 \%$ had a substance use disorder. Of these, $50 \%$ were diagnosed with an illicit substance use disorder, $70 \%$ were diagnosed with an alcohol use disorder, and $20 \%$ were diagnosed with both an illicit substance and alcohol use disorder. Alcohol use was reported by $64.3 \%$ of sexual minority persons (compared to $55.1 \%$ of the heterosexual population) and alcohol use disorders were reported by $12.4 \%$ of sexual minority persons (compared to $10.1 \%$ of the heterosexual population). Similar elevated prevalence of substance use compared to the general population were observed across nearly every substance domain. Substance use disorders in sexual minority persons may persist for longer with less intervention due to drinking and substance use norms within the sexual and gender minority community (Cochran, Grella and Mays, 2012), which may mean that upon presenting for treatment, sexual minority clients could have more severe symptoms and pervasive consequences from their substance use. In particular, among sexual minority men, stimulant use is much higher than among heterosexual men. Crystal methamphetamine specifically is much more commonly used by sexual minority men and is highly associated with sexual behavior that can lead to HIV acquisition and HIV transmission (Halkitis, Parsons and Stirratt, 2001; Mayer, Colfax and Guzman, 2006). Although it is the focal point for several research studies, including intervention studies, (Carrico et al., 2013; Carrico, Nation, et al., 2015; Carrico, Gómez, et al., 2015; Carrico et al., 2016, 2018; Mimiaga et al., 2012, 2019), community options for evidence-based and LGBTQ+ tailored treatment remain limited.

In sum, significant disparities exist across disorders for sexual minority individuals with few rigorously tested evidence-based LGBTQ+-affirmative and tailored interventions developed or available at a community level. Therefore, in each section that follows we review those treatments that have been specifically adapted and tested for LGBTQ+ clients as well as provide recommendations on how best to implement existing evidence-based practices in a way that is affirming of sexual minority clients.

\section{Treatment Utilization}

Sexual minority persons access mental health services more than heterosexual persons even when controlling for demographic variables, comorbidities, and differences in prevalence of disorders (Cochran, Björkenstam and Mays, 2017). Cochran and colleagues (2017) hypothesized this finding may reflect the use of professional mental health services to deal with other life stressors including those caused by minority stress (Cochran, Björkenstam and Mays, 2017). However, few clinicians are adequately prepared to provide affirming care (Rock, Carlson and McGeorge, 2010; Graham, Carney and Kluck, 2012; Farmer, Welfare and Burge, 2013). Given both higher frequency of mental health symptoms and higher levels of treatment seeking, it is imperative that there be specialized training for clinicians to provide affirmative interventions in practice. Accordingly, the goal of this chapter is to help inform culturally 
responsive interventions to address emotional trauma, and substance use disorders when working with sexual minority clients.

\section{Minority Stress Framework}

The minority stress framework (Meyer, 2003) offers one theoretical perspective from which to understand and interpret health disparities affecting sexual minority communities. According to the minority stress framework, individuals who identify as sexual minorities are exposed to elevated levels of stress due to LGBTQ+ specific societal, interpersonal, and internalized stigma. This excess stress is constantly present and can directly and/or indirectly lead to the disproportionate mental and behavioral health conditions including emotional and substance use disorders that are consistently observed among sexual minority communities.

As such, the minority stress framework is helpful for understanding why mental health disparities are present across disorders and how larger, systemic injustices including environmental policies, the sociopolitical climate, and interpersonal discrimination can elevate risk for all stress-sensitive mental health and substance use concerns. Of note, several of the efficacious interventions that have been tested specifically for sexual minority persons utilize the minority stress framework to inform intervention adaptations (Layland et al., 2020). The recommendations provided herein are based on the minority stress framework as we conceptualize existing disparities and interventions to address them as resulting from societal and social inequities that place sexual minority persons at increased vulnerability for stress-sensitive disorders.

\subsection{Interventions for Emotional Disorders}

To our knowledge, despite significantly higher prevalence of anxiety and depressive disorders among sexual minority individuals compared to their heterosexual counterparts, few evidencebased interventions have been specifically adapted to meet the needs of sexual minority clients. One strategy that can be used to deliver sexual minority-affirming treatment, particularly in the absence of available adapted interventions, is to apply existing non-adapted interventions with some considerations regarding the unique contexts in which sexual minority clients may be experiencing their presenting concerns. We offer some general considerations here for how best to implement existing, efficacious interventions with sexual minority clients.

\section{General considerations for treatment of emotional disorders in sexual minority persons}

Cognitive behavioral therapy (CBT) is widely documented as an efficacious intervention for anxiety disorders, obsessive compulsive spectrum disorders, and depressive disorders. Across these disorders, treatment involves psychoeducation, cognitive reappraisal and restructuring, and engagement in activities that had been previously avoided.

\section{Psychoeducation}

Following a CBT approach, information about an individual's psychological presentation is typically provided to a client within the first few treatment sessions. As part of that background information, therapists might address current symptoms, origins of symptoms, and how they 
interfere with daily living. For sexual minority clients, it may be useful to include psychoeducation about the minority stress framework and how experiences of minority stress may impact their symptoms (e.g. avoiding situations or experiences due to fears of being judged for their sexual orientation and/or gender expression). When delivering such psychoeducation, it is useful to strike a balance between providing information about what is known in the empirical and theoretical literature about the relationship between minority stress and psychological distress, while at the same time, collaboratively assessing the degree to which these relationships fit for an individual client.

\section{Cognitive reappraisal and restructuring}

With regard to cognitive appraisals, clients commonly experience maladaptive beliefs about a host of psychosocial stimuli (e.g., being disliked or fearing others having negative opinions of them). Through CBT sessions, the therapist encourages the client to "challenge" these inflexibly negative perceptions of themselves and to rate the likelihood that such beliefs are true.

When working with sexual minority clients, it is important to consider the possibility that fears of being disliked because of one's sexual orientation identity could be accurate, due to the reality of interpersonal stigma in some sexual minority clients' lives. Therapists who do not consider this possibility may not be fully aware of the reality of many sexual minority clients' lives, and as such, may be dismissing important, reality-based concerns that clients are expressing. In these contexts, it is more useful to focus on challenging the core beliefs that sexual minority clients may hold (e.g., "I am unlovable," "I am worthless," or "I am a failure") that are certainly inaccurate even if there are anti-LGBTQ+ people or institutions (e.g., religious organizations, schools, etc.) in their lives who have contributed to these beliefs and caused sexual minority clients harm.

When working with sexual minority clients it is important to know and understand their backgrounds and experiences around their sexual orientation identity to fully appreciate which beliefs may be accurate appraisals of a specific situation. For negative yet accurate appraisals (e.g., being rejected by family members who do not accept their sexual orientation), acceptance-based strategies (e.g., asking "how helpful is this thought?") or problem-solving techniques may be useful for navigating these situations. Cognitive defusion techniques that help clients distance themselves from their thoughts could also be useful (e.g., singing a negative thought, saying it in different voices, writing it down repeatedly until it loses its "power," etc.).

\section{Engagement in previously avoided activities}

Similarly, behavioral exposures (in the case of anxiety disorders and obsessive-compulsive disorders) and increased activities (in the case of depressive disorders) are intended to help an individual engage in previously avoided activities. However, for sexual minority clients, it is possible that some exposures could pose a risk to their safety. For example, fearing discrimination while having a romantic dinner at a restaurant with a same-sex partner may be realistic and should be approached with an appreciation of the context in which a client lives. In 
some cases, clients may live in environments where such an exposure could pose a safety risk due to the anti-LGBTQ+ attitudes and behaviors of others. We recommend carefully and collaboratively assessing the actual risk during the exposure given the individual's environment. Safety should always be the priority in planning exposures. On the other hand, it is possible that feared situations may be safe and excessive concerns may be targets for intervention. For instance, although a client may fear that others would judge them while eating a romantic dinner with their partner at a restaurant, in some settings, it may objectively be a safe exposure in which the client might learn how to habituate to anxiety-causing situations in order to pursue valued activities with less difficulty. A careful functional analysis early on in treatment can help guide decisions regarding exposure hierarchies, behavioral experiments, and engagement in previously avoided activities.

\subsection{Interventions for Substance Use Disorders}

Many studies testing interventions to address substance use disorders that are tailored for sexual minority clients have focused on sexual minority men and HIV prevention or improving health among those living with HIV. We provide a brief overview of those interventions here. Far fewer have been conducted among sexual minority women despite significant disparities. However, it is possible that some of the interventions described below could be adapted or tested with sexual minority women, as investigators continue to build this area of clinical research and funding for such work becomes increasingly available.

\section{Motivational interviewing approaches}

Motivational interviewing (MI) (Miller and Rollnick, 2012) is a style of counseling that can be used to explore ambivalence for individuals who are experiencing some negative consequences from their substance use, but may not be ready to change their current behaviors. MI is a clientcentered approach that is particularly helpful in guiding clients through their own exploration of the costs and benefits of their substance use.

MI has been adapted for use with sexual minority men to address substance use disorders and sexual behaviors associated with HIV transmission among HIV negative men as well as men living with HIV. One study by Parsons and colleagues involved an 8-week trial of MI sessions for addressing substance use and HIV medication adherence in sexual minority men and found that $\mathrm{MI}$ sessions led to a significant decrease in substance use and a trend towards significance in improved antiretroviral medication adherence (Parsons et al., 2005). The same research group conducted a trial several years later (2014), titled the "Young Men's Health Project," which compared four sessions of $\mathrm{MI}$ to four sessions of an education control condition to address substance use and sexual risk among young gay and bisexual men. This study found that $\mathrm{MI}$ was superior to the education condition as men who received MI were less likely to use drugs and engage in condomless anal sex (Parsons et al., 2014).

MI has also been used in partner interventions for substance use among sexual minority men. In a trial of three Ml sessions with sexual minority male couples, use of $\mathrm{Ml}$ in dyads where at 
least one partner was using substances was effective in improving relationship functioning (Starks et al., 2018, 2020).

\section{Contingency Management}

Contingency management is an efficacious intervention for substance use treatment and has been used successfully with sexual minority men who use methamphetamines in clinical trials and in community settings. Contingency management provides positive reinforcement in the form of tangible incentives for abstinence (Prendergast et al., 2006) and has been shown to be effective for the treatment of methamphetamine use in sexual minority men when used in isolation (Reback et al., 2010) and when combined with CBT (Shoptaw et al., 2005). Further, it has been shown to enhance substance use disorder treatment (Roll et al., 2006). Contingency management in combination with urine-screening visits, drop-in group support, and referral for other services in a community setting was a feasible and acceptable form of substance use treatment for methamphetamine using men who have sex with men (MSM) (Gómez et al., 2018). However, in some cases contingency management did not outperform the control condition; therefore, contingency management alone is unlikely to be a successful intervention for reducing crystal methamphetamine or other substance use in general or with sexual minority clients (Shoptaw et al., 2005; Menza et al., 2010).

The Matrix Model: Cognitive Behavioral Therapy, Motivational Interviewing, and Contingency Management

The Matrix Model (Rawson et al., 2004) is an evidence-based cognitive behavioral outpatient substance use disorder treatment program. The Matrix Model has been proven effective in the treatment of stimulant use disorders and has been adapted specifically for use with sexual minority men. The most appropriate therapist to deliver this treatment will be well trained in MI, CBT, and addiction neuroscience and will be able to flexibly respond to content in treatment sessions. The matrix model has 8 guiding principles: (1) Building strong and respectful relationships between therapist and client, (2) Maintaining consistency and following through on the structure of the program and expectations of the client, (3) Providing quality educational content for the client on the scientific nature of substance use, (4) Using varied treatment approaches - including CBT and MI - to meet the client's needs, (5) Rewarding and encouraging desired behaviors while extinguishing negative behaviors, (6) Engaging and educating family about addiction and recovery, (7) Motivating members to participate in self-help and community based programs, (8) Checking for substance use with urine testing (Rawson et al., 2004).

The Matrix Model has been adapted for use with sexual minority men seeking treatment for methamphetamine use by Shoptaw and colleagues (Shoptaw et al., 2005) and showed moderate effects in abstinence from stimulants, which were associated with CBT and contingency management. Another trial comparing this culturally tailored Matrix Model to a gay social support group found that the Matrix Model treatment was superior on substance use outcomes (Shoptaw et al., 2008). This Matrix Model treatment has been utilized in other clinical research with a focus on harm reduction; and, notably, has been associated with a reduction in 
stimulant use, addiction severity, and condomless anal intercourse with the potential for HIV transmission even when implemented in community settings (Carrico, Gómez, et al., 2015).

\section{Positive Affect Interventions}

Other interventions have focused on addressing positive and negative affect in sexual minority men who use substances, particularly crystal methamphetamine, based on data to support the importance of positive affect in active coping and self-efficacy in avoiding triggers and remaining abstinent (Carrico et al., 2013). Carrico and colleagues tested positive affect interventions in community settings in San Francisco (Carrico et al., 2013) and in randomized trials for sexual minority men living with HIV who also use crystal methamphetamine and noted significant improvements in positive affect and ART medication adherence compared to a neutral writing intervention (Carrico et al., 2019).

\section{Behavioral Activation}

Behavioral activation has been studied extensively for use with men who have sex with men and use stimulants. Theoretically, a BA approach involves increasing clients' engagement in activities that are enjoyable, replacing substance use, and helping individuals re-engage in meaningful life activities (Mimiaga et al., 2018).

Mimiaga and colleagues have tested behavioral activation in combination with sexual risk reduction for addressing stimulant use in men who have sex with men. Behavioral activation compared to control treatment led to a significant decrease in crystal methamphetamine use, depressive symptoms, and other substance use in a pilot trial (Mimiaga et al., 2012). Similar results were found in the initial randomized controlled trial results (Mimiaga et al., 2019), suggesting that BA may be an effective approach for managing methamphetamine use and anhedonia symptoms of depression in men who have sex with men.

\section{General considerations for treatment of substance use disorders in sexual minority clients}

The interventions described above were tailored to meet the needs of sexual minority clients. For sexual minority men, these interventions have largely focused on alcohol and methamphetamines (and other "party" drugs) and how they affect sexual health. Understanding the function of substance use within the community is important when delivering these interventions. Substance use can be a developmentally typical part of teenage and early adulthood for all communities, and in particular for sexual minority persons for whom social support and connection with other members of the LGBTQ+ community are often embedded in social and physical spaces where alcohol and substances are present and consumed. Clinicians should be careful to not over pathologize substance use to the extent that it is not causing problems, as doing so may be dismissive of the pervasive presence of alcohol in settings where sexual minority clients often find support. Historically, safe spaces for the LGBTQ+ community, and specifically, sexual minority men, have been in bars and clubs, spaces where alcohol and drugs are consumed. This is especially important to consider when working with sexual minority clients as in many cities affirming spaces are within the club and 
bar scene, limiting other spaces for congregating with friends or meeting partners (Easton et al., 2007; Garcia et al., 2015; Rogers et al., 2019).

Given the importance of developing positive social connections and activities, clinicians should mindfully balance the need for these positive social experiences with the reality of substances being present in many of these social settings. Working collaboratively with clients can help to ensure that they make plans that are congruent with their own goals. For example, for a sexual minority man who is using crystal methamphetamine during casual sexual encounters he finds on hook-up apps, it may be reasonable to suggest discontinuing the use of hook-up apps to meet partners, but not reasonable to avoid going to bars or social events where there may be alcohol or drugs present if this is the only setting for finding LGBTQ+ affirming social connections. Similarly, for a single sexual minority woman who is trying to reduce her problematic alcohol use, it may be reasonable to suggest meeting dates over dinner instead of "for a drink," but it may not be reasonable to encourage avoiding social events where alcohol is present altogether given the paucity of such affirming events in many areas of the U.S.

Finally, although Alcoholics Anonymous (AA) and Narcotics Anonymous (NA) are common sources of support for persons in recovery, clinicians working with sexual minority clients should collaboratively consider the possibilities of such resources being helpful and/or harmful. Both organizations ask individuals to seek a "higher power" for help in their recovery and have historically not always been welcoming of the LGBTQ+ community (Sanders, 2020). Although some groups may have a flexible and open interpretation of that meaning (e.g., love for humanity), there are other groups that are guided by a more religious interpretation. When working with individuals who use substances, it could be worth exploring local meeting groups and learning more about their philosophies. Notably, there are groups that explicitly state that they are affirming spaces and welcome members of the LGBTQ+ community. For clients who are interested in pursuing support from 12-step recovery meetings, we recommend making referrals to those groups that are explicitly affirming. It is not uncommon for sexual minority clients to have had negative experiences with religion and religious organizations. Overall, it is important to consider the religious history and experiences of sexual minority clients and collaboratively explore the degree to which AA and NA meetings, particularly those embedded in a more religious interpretation, could be beneficial versus harmful to the client. This may help facilitate clients making their own decisions about their recovery support options.

\subsection{Interventions for Trauma}

Sexual minority persons are significantly more likely to have experienced trauma than the general population. Experiences of concealment of sexual identity and discrimination can be traumatic and/or lead to trauma. Additionally, sexual minority persons report higher rates of childhood sexual abuse, a known correlate of adult sexual assault (Friedman et al., 2011). Compounding experiences of trauma is the fact that same-sex sexual trauma is often unreported (Stemple and Meyer, 2014; Juyal et al., 2017). Without the appropriate channels to seek justice for these traumas, individuals may experience increased trauma-related stress. For individuals experiencing trauma related to experiences of concealment, discrimination, or some combination of factors, it is important to normalize and destigmatize these experiences to 
bolster resiliency. Notably, most individuals who experience traumatic events do not develop post-traumatic stress disorder (PTSD) (Breslau, 2009). However, in some cases, trauma responses may not resolve on their own and instead progress to include symptoms of PTSD (e.g., recurrent intrusive thoughts, nightmares, hypervigilance, and avoidance as well as a negative view of the world and self that persists beyond an acute period). In these cases, it is important to provide evidence-based trauma interventions to address these symptoms.

Perhaps the most widely tested CBT treatments are prolonged exposure (PE) and cognitive processing therapy (CPT). Each incorporates, to a different extent, elements of

psychoeducation, skills training, exposure, and cognitive restructuring. PE relies more heavily on exposure, and specifically, the use of imaginal exposure, to recall and relive the traumatic event through remembering and recanting specific details. The theoretical basis for this approach is that by narrating the trauma the individual can help organize the memory and distinguish between the memory and the trauma. The process of retelling the trauma also helps the client to tolerate distress associated with the memory and increase confidence in the ability to manage associated distress (Foa, Hembree and Rothbaum, 2007; Foa, 2011). In contrast, CPT relies more heavily on cognitive restructuring to address the negative beliefs that clients have about themselves, others, and the world following a traumatic event. In CPT, the goal is to evaluate the impact of a trauma (through an impact statement written by the client) and to encourage the client to examine how beliefs about themselves and the world changed as a result of the trauma (Resick and Schnicke, 1993).

Despite the high occurrence of traumas in sexual minority populations, there are a few PE, CPT, or other CBT-based trauma interventions that have been specifically tailored to sexual minority clients and focus on experiences of trauma (e.g., sexual trauma) that may be especially salient to one's sexual orientation identity. Specifically for sexual minority men, O'Cleirigh and colleagues (2019) designed and tested a cognitive behavioral therapy and trauma self-care (CBT-TSC) intervention for addressing childhood sexual abuse, sexual compulsivity, and sexual health behaviors in sexual minority men reporting behavior that could lead to HIV acquisition (O'Cleirigh et al., 2019). Trauma treatment components were based in CPT and tailored to be affirming of sexual minority men and their sexual behavior and specifically explored relationships between sexual trauma history and current sexual behavior. Compared to voluntary counseling and testing, which is the standard of care for HIV prevention, participants who received CBT-TSC reported significantly fewer instances of condomless anal sex following the intervention as well as significantly fewer avoidance symptoms of PTSD. To date, there are no published results to the authors' knowledge for trauma-specific interventions specifically for use with sexual minority women.

\section{General considerations for treatment of trauma in sexual minority persons}

There is a high occurrence of childhood sexual abuse among sexual minority individuals compared to heterosexual persons (Bailey et al., 2016). Some sexual minority clients may believe a myth that their non-heterosexual sexual orientation was "caused" by sexual abuse earlier in life. There is no causal relationship between childhood sexual abuse and adult sexual orientation (Saewyc, 2011; Bailey et al., 2016). Early researchers falsely suggested that 
childhood sexual abuse led to the development of a sexual minority identity in reaction to that abuse (Simari and Baskin, 1982), further perpetuating these beliefs within the general public. Saewyc (2011) articulates the illogical nature of this argument, which may be particularly useful for therapists to understand when working with clients who hold this belief about themselves (Saewyc, 2011). For example, in the majority of sexual abuse cases, the abuser is male identified; therefore, the myth that being abused by men leads women to seek female partners whereas it leads men to seek male partners is logically inconsistent. They further argue that if childhood sexual abuse causes one to later have a sexual minority identity, then the majority of sexual minority identified individuals would report a history of childhood sexual abuse. Despite the higher prevalence of childhood sexual abuse among sexual minority individuals, it is clearly not the case that all sexual minorities, or even a majority, have experienced this traumatic experience. Instead, the disparity in childhood sexual abuse is believed to be a result of abusers identifying children who may already be more lonely, isolated, or otherwise vulnerable based on their social environment and positionality. It is important, as a clinician, not to collude with myths about childhood sexual abuse being causally related to a sexual minority identity. One strategy for not colluding could be to share the above information with clients in a clear and affirming way. For example, "This is a belief that clients sometimes have. Is it okay if I share some information with you about the relationship between sexual abuse and sexual orientation?" With the client's permission the therapist could offer, "I want you to know that there is no scientific evidence to support this. How does this information impact the way you view yourself and your sexual identity?"

Another consideration is the degree to which sexual orientation was a central factor in sexual minority clients' experiences of abuse. Some sexual minority clients may have experienced verbal and/or emotional abuse from parents or caregivers who tried to "change" their sexual orientation or behaviors that they perceived to be associated with their child's sexual orientation. For example, a parent finding their child playing with toys prescribed for children of another gender (e.g., boy playing with dolls, girl playing with toy trucks) may punish this behavior through verbal commands and/or physical forms of abuse. In other cases, sexual minority clients may have been exposed to verbal, emotional, or physical abuse by parents or caregivers unrelated to their sexual orientation identity or gender expression. However, in other cases, such abuse may be directly tied to parental disapproval of their expressions of their sexual orientation or gender identity. It can be helpful to explore the connections between the clients' experiences of abuse and their sexual orientation/gender expression to understand what types of interpretations the client may have about their abuse. Clients may realize that these early experiences continue to impact their feelings about their sexual orientation identity or aspects of their gender expression currently, including experiencing internalized stigma or shame. Recognizing the role these early experiences have had in their understanding of self can be a starting point for healing.

Additionally, sexual minority persons, particularly those whose gender expression may not conform to prescribed gender roles, may have been exposed to a range of bullying experiences, from verbal all the way to extreme physical bullying from peers as children. In these cases, clients may perceive their sexual orientation identity and/or gender expression to have caused their traumatic experiences. Importantly, it is not their identity or expression, but the anti- 
LGBTQ+ responses of others to it that caused these events. These early experiences may still be influencing how a client responds to conflict and it is important to explore the ways in which this early social feedback may have led to high levels of rejection sensitivity, approval seeking, and/or unassertive behaviors in adulthood. For example, a therapist might offer an interpretation such as, "You had a hard time fitting in then. I wonder how that has affected how you feel when being with groups of people now. Tell me about what that is like for you."

Finally, everyday experiences of discrimination, particularly when they are repeated and chronic, may generate trauma symptoms (Kaysen, Lehavot and Dworkin, 2019). Similar assertions have been made of trauma experienced individuals who are ethnic/racial minorities (Jernigan et al., 2015); and, although we focus on sexual minority specific literature, it is important to note that sexual minority individuals exist within other communities, which impact the level of minority stress an individual experiences and how that intersects with other forms of minority stress. An individual with multiple marginalized identities is likely to also be at risk for several stress-sensitive emotional conditions. For example, there are documented differences in the prevalence of mood, anxiety, and substance use disorders among sexual minority persons when stratified by race/ethnicity (Rodriguez-Seijas, Eaton and Pachankis, 2019). It is important for the clinician to consider intersecting systems of oppression, which place the client at higher risk of experiences of other forms of marginalization and how they intersect with sexual minority status within the clinical sphere. Examples of "everyday trauma" that sexual minority clients may experience include an anti-LGBTQ+ comment from a bystander while holding hands with a same-sex partner, seeing a news headline about discrimination against sexual minority persons, or having to indicate on a form with heteronormative options that their spouse is a same-sex partner. These daily experiences can manifest into trauma symptoms (e.g., hypervigilance, negative views about self and the world, intrusive thoughts, etc.) even in the absence of a Criterion A trauma and should be addressed within the course of treatment.

\subsection{Interventions for Disordered Eating and Body Dissatisfaction}

Efficacious treatments exist for improving body image and disordered eating in both group and individual settings (Hay, 2013). Treatments for the prevention and/or early intervention of body image and eating disorders largely fall into dissonance-based interventions (Stice et al., 2012, 2015; Stice, Yokum and Waters, 2015) or CBT based interventions. Dissonance based interventions, which encourage clients to challenge beliefs about an ideal body image (e.g., thin ideal, muscular ideal) through speaking and acting against these beliefs to create cognitive dissonance, have been tested primarily in college aged women without specific attention to sexual orientation and in group settings for the prevention of eating disorders. Although efficacious, these will not be a primary focus of this section because they typically address nonclinical symptomatology that falls within the normative range. However, it is important to note that one such intervention, The Body Project, was adapted to reduce eating disorder risk for sexual minority men and was found to be efficacious. This version of The Body Project( was titled, The PRIDE Body Projecic and aimed specifically at reducing risk factors for disordered eating among gay men in a university setting (Brown and Keel, 2015). The project recruited 87 males who were assigned to either a 2-session dissonance-based intervention or a waitlist control condition. Participants completed assessments at baseline, post-intervention, and 4- 
week follow-up. The intervention was rated as highly acceptable and the dissonance-based condition led to significant decreases in measures of body dissatisfaction, drive for muscularity, self-objectification, body-ideal internalization, dietary restraint, and bulimic symptoms (e.g., bingeing and purging behavior). These improvements were maintained at follow up, except for body ideal internalization, showing sustainability of treatment effects.

The same authors also modified this dissonance-based program to address concerns about muscularity in sexual minority men in a program titled The Body Project: More than Muscles@ (Brown et al., 2017). This program differed from the first in that it specifically addressed concerns associated with a "muscular ideal." The program was highly acceptable among sexual minority men and the dissonance-based condition led to decreases in body-ideal internalization, dietary restraint, and bulimic symptoms. In this project, they also specifically measured drive for muscularity and muscle dysmorphia symptoms, since that was a target of the intervention, and found significant improvements in these symptoms as well compared to the waitlist control condition. All treatment gains were maintained at one-month follow-up. Whereas prior interventions were designed for women and focused on the "thin ideal," this intervention was focused on the "muscular ideal." This is an important distinction because disordered eating in men (including sexual minority men) may be informed by gendered expectations of ideal body size and shape. As such, disordered eating among men tends to include habits like replacing meals with protein smoothies, counting carbohydrates, excessive exercise, and use of supplements. In contrast, eating behaviors that may be more often discussed as features of disordered eating include counting calories, fasting, and avoiding foods containing fat. These behaviors, primarily observed among women with disordered eating, are also informed by gendered expectations of ideal body size and shape (Blashill, Brown and Klimek, 2019).

Among CBT interventions and third-wave CBT interventions, many different approaches have been tested in RCTs and show efficacy for the treatment of eating disorders. These include enhanced cognitive behavioral therapy, or CBT-E, a transdiagnostic eating disorder intervention (Fairburn, Cooper and Shafran, 2003), family behavior therapy for eating disorders in adolescents (Le Grange and Lock, 2007; Lock and Le Grange, 2013), dialectical behavior therapy for bulimia nervosa (Safer, Telch and Agras, 2001; Safer, Couturier and Lock, 2007), and interpersonal psychotherapy (IPT) for binge eating disorder (Wilfley et al., 2002). To our knowledge, these interventions have not been tested specifically for sexual minority populations. They have included women and men and likely included sexual minority participants, however, data on sexual orientation of participants was not published. Below, we provide some general considerations for tailoring CBT-based interventions for sexual minority clients. Any of these recommendations can be applied to DBT or IPT as well, with specific attention to how the psychosocial and broader sociocultural context may affect distress tolerance (addressed in DBT) and/or interpersonal functioning (addressed in IPT).

\section{Enhanced cognitive behavioral therapy for eating disorders: CBT-E}

For the purposes of this section of the chapter, we focus on adaptations to CBT-E (Fairburn, Cooper and Shafran, 2003) because it is a transdiagnostic approach that highlights the core processes underlying eating disorders and can be applied in clinical practice regardless of the 
specific features of the disordered eating behavior. As a transdiagnostic approach, it may also apply to those with subthreshold conditions for whom disordered eating is still their presenting concern.

In addition to an over evaluation of weight and shape, core clinical features of eating disorders include: (1) clinical perfectionism, (2) mood intolerance, (3) core low self-esteem, and (4) interpersonal difficulties (Fairburn, Cooper and Shafran, 2003). For sexual minority clients who have been undervalued as a result of stigma and discrimination based on their sexual orientation identity, it is common to experience core beliefs reflecting low self-esteem. Relatedly, sexual minority clients may be more likely to emphasize external contingencies of self-worth, along with feeling immense pressure to achieve in those domains, which in turn leads to clinical perfectionism. This phenomenon has been termed the "best little boy in the world hypothesis" when applied to young sexual minority men (Pachankis and Hatzenbuehler, 2013) and describes a pattern of behavior by which someone tries to be "perfect" in all other ways to "compensate" for perceived inadequacies as a sexual minority person. Congruent with the key clinical features of eating disorders, sexual minority clients may, due to the impact of antiLGBTQ+ stigma, experience interpersonal difficulties resulting from concealment of sexual orientation (Pachankis, 2007) or unassertiveness in interpersonal contexts.

As such, addressing the underlying core psychopathology of eating disorders, as is suggested by the CBT-E model, may also address patterns of behavior attributable to sexual minority stress. Important considerations would be the way in which sexual minority clients may over evaluate their weight and shape (e.g., for sexual minority men striving to fit in with a muscular ideal within the community) or experience dysregulated eating (e.g., avoiding carbohydrates) in order to achieve unrealistic body standards that may uniquely impact sexual minority clients due to external contingencies of self-worth (Blashill, Brown and Klimek, 2019). Importantly, therapists should take a client-centered approach to understanding their clients' body ideals and preoccupations rather than imposing gendered expectations on what these body ideals may be, as they do not always align with rigid gender roles.

A recommended course of CBT-E would be approximately 20 sessions over 20 weeks (Fairburn, Cooper and Shafran, 2003). Following CBT-E, the case formulation identifies the core psychopathology that is maintaining over evaluation of weight and shape concerns and contributing to disordered eating, purging, dieting supplements, or other weight control behaviors. For sexual minority clients, it may be useful to explore how one's experiences as a sexual minority person may contribute to maintaining the eating disorder psychopathology. For instance, holding high external contingencies of self-worth based on physical appearance could be linked to experiencing rejection based on one's sexual identity. Related, seeking to achieve impossible body standards could be linked to unrealistic expectations within the gay community. Importantly, we encourage therapists to take a client-centered approach to understand their clients' body ideals and preoccupations, rather than imposing gendered expectations on what these body ideals may be. There may be differences in "body ideal" for clients who align with various sexual minority subcultures and communities that differ from the "thin ideal" often described in the eating disorder literature. Ideals based on sexual minority subcultures to which individuals belong or aspire could exert differing effects on individuals' body ideals and images. 
Therapists can implement self-monitoring of eating and purging behavior, encourage regular eating at mealtimes to avoid bingeing behavior (thought to develop from extreme restriction), and in-session weighing to monitor progress. The final stage is building skills for relapse prevention including being aware of environmental triggers (e.g., comparing self to others in social settings) and internal triggers (e.g., feeling unlovable) to avoid slipping back into disordered eating habits.

\section{Body Dysmorphic Disorder}

Eating disorders and subclinical or associated pathology typically include a component of body dissatisfaction. However, when that dissatisfaction becomes the presenting concern and manifests in a way that is distinct from weight and shape concerns only, it may be more appropriate to conceptualize the individual as experiencing Body Dysmorphic Disorder (BDD).

As a point of distinction, BDD is characterized by a preoccupation with an imagined defect in appearance. Common preoccupations include concern over skin, hair, or a specific body part (e.g., nose, chin) and thoughts are focused on this problem for at least one hour a day. This preoccupation also causes clinically significant distress and impairment (Bjornsson, Didie and Phillips, 2010; 'Obsessive-Compulsive and Related Disorders', 2013).

Wilhelm and colleagues developed a modular cognitive behavioral therapy for BDD (CBT-BDD) that has been shown to reduce BDD severity (Wilhelm et al., 2014). This modular treatment includes psychoeducation, cognitive interventions to address maladaptive thinking including the overemphasis on the importance of appearance for self-worth, exposure exercises, mindfulness, and perceptual retraining (to see the gestalt rather than details of appearance since bottom-up processing is thought to contribute to body dysmorphia symptoms).

Similar to adaptations for eating disorders, it may be beneficial for clinicians to explore the origin of sexual minority clients' thoughts around image and appearance as related to other feelings of low self-worth. For example, sexual minority clients may have internalized inaccurate, antiLGBTQ+ messages that they are "defective" or "damaged" in some way and a focus on a specific body part may reflect those negative feelings about oneself. As such, with sexual minority clients it may be helpful to explore the origins of these negative feelings about oneself by using a sexual minority stress framework and considering the impact of societal messages about appearance and self-acceptance as it relates to sexual orientation identity.

Body image disturbance, or clinical features resembling BDD, but meeting full criteria (e.g., lesser impairment, present fewer days), may also benefit from similar interventions and we would recommend a similar approach to treatment. There has been one intervention that has been specifically adapted to address body image disturbances in sexual minority men living with HIV who may be especially prone to concerns about their appearance due to body changes that occur from medication taken to treat HIV, specifically antiretroviral therapy (ART). This intervention, designed by Blashill and colleagues (2017) dually addressed body concerns and medication non-adherence for sexual minority men living with HIV (Blashill et al., 2017). 
The CBT Body Image and Self-Care or CBT-BISC intervention is 12 total sessions divided among seven modules. Module 1 includes an orientation to the treatment and CBT model of body image disturbance; Module 2 focuses on mindfulness and acceptance-based strategies; Module 3 focuses on perceptual retraining, during which participants complete a "mirror exposure" to try to see a gestalt rather than focus on specific parts of their body and includes mindfulness strategies; Module 4 introduces cognitive restructuring; Module 5 includes more invivo exposure strategies as well as exposure to situations that are avoided; Module 6 focuses on response prevention (or avoiding ritualistic behavior); and Module 7 includes relapse prevention skills (Blashill et al., 2017). In a two-arm randomized controlled trial compared to enhanced treatment as usual, CBT-BISC led to a reduction in body image disturbance and depression and improvements in medication adherence and global functioning that were maintained at 3-months post-intervention. This suggests that: (1) BDD treatment can be applied in individuals with symptomatology similar to BDD, but not the full disorder with similar improvements and (2) modifying this treatment for sexual minority clients and their specific concerns (including other coexisting health conditions) can be effective.

General considerations for treatment of eating and body image disturbance in sexual minority clients

In general, when working on eating and body image disturbance with sexual minority clients it is important to consider how a sexual minority client's early and ongoing experiences with stigma and discrimination may relate to features of disordered eating behaviors and patterns.

Importantly, sexual minority clients have often received inaccurate societal messages about the inferiority of their identity compared to heterosexual individuals and may have internalized those anti-LGBTQ+ messages. These internalized messages about inferiority may contribute to feelings of low self-esteem and a self-worth that is overly reliant on measurable achievements or outcomes, including an ideal shape and weight. Conversely, when sexual minority clients who do experience low self-worth or contingent self-worth perceive a "failure" in one area of life, this may further reinforce the notion that body size and shape is a more controllable aspect of self that can be changed to meet external ideals. An interpersonal disagreement may lead to feelings of "no one will ever love me" and, in a person with distorted weight and shape concerns, this may be extended to "no one will ever love me because I am too fat/not muscular enough." It is also important to consider the role social environments may have on reinforcing these beliefs. For example, dating applications, particularly those used by sexual minority men, can reinforce existing body image concerns (Tran et al., 2020). Clinicians should be mindful of addressing the presenting psychopathology as well as exploring how sexual minority stigma and sexual minority community stress can contribute to these beliefs.

\subsection{Interventions Across Diagnostic Categories: Transdiagnostic Interventions}

Thus far, we have primarily reviewed intervention approaches in a disorder domain-specific manner, largely congruent with the typical approach to psychiatric and intervention science. However, findings from psychiatric nosology (Eaton et al., 2015; Kotov et al., 2017) and intervention science (Barlow, Allen and Choate, 2004; Farchione et al., 2012; Barlow et al., 
2017) point to the importance of attention to common processes underlying various diagnostic categories and forms of psychosocial distress.

Psychiatric symptoms do not occur in isolation among sexual minority persons, or most people for that matter. Instead, they operate in a synergistic manner to compromise sexual minority individuals' psychosocial wellbeing (Pachankis, 2015). As mentioned, several of the psychosocial concerns that arise for sexual minority clients occur across diagnostic categories and symptom expressions. Therefore, directing treatment toward the multiple co-occurring psychosocial processes underlying health disparities among sexual minorities aligns with recent empirical and clinical advances toward a transdiagnostic approach within the general population, which similarly targets shared psychosocial mechanisms that underlie most mental health disorders.

The Unified Protocol was developed as a transdiagnostic intervention focused on the processes common across mood and anxiety disorders (Barlow et al., 2017; Sauer-Zavala et al., 2020). It has since been adapted to formally address the co-occurring mental, behavioral, and sexual health concerns of sexual minority men and women. The Effective Skills to Empower Effective Men (ESTEEM) and Empowering Queer Identities in Psychotherapy (EQuIP) protocols were developed through systematic consultation with key stakeholders (Pachankis, 2014; Pachankis et al., 2020).

Both interventions are based on key CBT-related intervention principles described above, with explicit direction for exploration of the ways that sexual minority stress is related to learning mechanisms that predispose and maintain mental health, substance use, and sexual health concerns. The treatments are underscored by six major treatment principles (Pachankis, 2014):

1. Normalize emotional and behavioral responses to minority stress.

2. Challenge maladaptive thoughts largely in line with traditional CBT-based approaches, with the necessary considerations for tailoring such intervention to sexual minority persons.

3. Increase and empower assertive communication patterns.

4. Validate sexual minority persons' individual and collective strengths.

5. Affirm and normalize sexual minority clients' sexual experiences and expressions.

6. Facilitate the formation and strengthening of supportive relationships.

The ESTEEM (Pachankis et al., 2019) and EQuIP (Pachankis et al., 2020) interventions both consist of 10-session protocols and are predicated upon these six core principles. Therapists proceed through the treatment modules, adapted from the Unified Protocol, with attention to the ways in which minority stress compromises psychosocial health in relation to the above core principles.

There is preliminary evidence available for both interventions. For ESTEEM, the initial pilot treatment significantly reduced depressive problems, alcohol use problems, sexual compulsivity, past-90-day condomless sex with casual partners, and increased condom use self-efficacy; and, 
the effects of the intervention were maintained at follow-up (Pachankis et al., 2015). Results from the randomized-controlled trial are forthcoming; and the protocol, methods, and design can be referenced here (Pachankis et al., 2019).

For EQuIP, compared to the waitlist condition, participants who received the EQuIP intervention experienced significantly reduced depression and anxiety and reduced alcohol use. Minority stress processes were also affected, indicating that the treatment functioned through addressing minority stress, as hypothesized (Pachankis et al., 2020).

\subsection{Future Research Directions}

Despite the many interventions that have been developed, more interventions focused on the integration of specific mental health conditions with tailored sexual minority content are needed. Specifically, although there are clinicians in the community working with sexual minority women and adapting evidence-based treatments to meet their specific needs, there are a paucity of published intervention trials establishing their efficacy and effectiveness, leading to a literature that is skewed towards focusing on the mental health of sexual minority men, most of which are in the context of HIV.

Future research should also address implementation of these interventions in community settings and evaluation of their effectiveness in community contexts. As is the case with other evidence-based treatments, once implemented in "real-world" settings, interventions may not be as feasible as they were in laboratory/research settings. Additionally, amongst those providers already working with sexual minority clients, there may be challenges with adopting new treatment approaches, even if shown to be effective. Similarly, it will be important, as new interventions are developed for testing in clinical trials, to continue to inform this work with "practice-based evidence" to ensure that the expertise and experience of those doing this work is integrated into clinical guidelines.

\subsection{Clinical Applications and Recommendations}

It is important to consider the ways in which treatment approaches could be adapted to better meet the needs of sexual minority clients. First and foremost, we encourage those who are working with sexual minorities to consult the guidelines from the American Psychological Association, APA Task Force on Psychological Practice with Sexual Minority Persons (APA Task Force on Psychological Practice with Sexual Minority Persons, 2021). Additionally, there are APA guidelines that are forthcoming specific to gender diverse clients, which we recommend be consulted if working with that client population, although many of the same principles apply.

Within this section, we provide a summary of the guidelines here as well as detailed clinical recommendations based on the extant literature and our own clinical experiences working with sexual minority clients. When possible, we recommend using evidence-based interventions tailored for sexual minority populations, as available. When not available, we recommend using

evidence-based interventions, but to inform clinical adaptations based on an appreciation of the 
unique experiences sexual minority clients may have-based on the minority stress framework (Meyer, 2003)_and maintaining a clear LGBTQ+-affirmative stance in psychotherapy as described in the transdiagnostic treatment approaches and adaptations above. Additionally, the following approaches are recommended for clinicians to provide evidence-based and affirming care.

\section{American Psychological Association Guidelines}

The American Psychological Association (APA) has guidelines for working with LGBTQ+ clients (APA Task Force on Psychological Practice with Sexual Minority Persons, 2021). These guidelines are updated periodically and are reviewed by experts in the field to ensure that they are culturally responsive and clinically appropriate. We provide a brief description here and encourage readers to access the guidelines for a more detailed explanation. The guidelines delineate 16 principles for practice in five domains as described here. Although published by the American Psychological Association to guide psychologists, these could be extended and applied to anyone who conducts clinical work with sexual minority persons including psychiatrists, social workers, mental health counselors, marriage and family therapy counselors, and peer support specialists. We encourage clinicians to review the outline below and to consult the guidelines for specific guidance on how to implement these approaches in practice.

\section{$\underline{\text { Foundational Knowledge and Awareness }}$}

This area of competency includes the following guidelines around developing basic knowledge and awareness of the identities of sexual minority persons and how they may intersect with other identities and experiences. It includes the following guidelines: (1) Psychologists understand that people have diverse sexual orientations that intersect with other identities and contexts, (2) Psychologists distinguish issues of sexual orientation from those of gender identity and expression when working with sexual minority persons, (3) Psychologists strive to affirm bi+ identities and examine their monosexist biases, (4) Psychologists understand that sexual minority orientations are not mental illnesses and that efforts to change sexual orientations cause harm.

\section{$\underline{\text { Impact of Stigma, Discrimination, and Sexual Minority Stress }}$}

This domain includes guidelines on how to approach the experiences of stigma and discrimination and the impact that has on sexual minority stress, wellness, and resiliency. It includes the following guidelines: (5) Psychologists recognize the influence of institutional discrimination that exists for sexual minority persons and the need to promote social change, (6) Psychologists understand the influence that distal minority stressors have on sexual minority persons and the need to promote social change, (7) Psychologists recognize the influence that proximal minority stressors have on the mental, physical, and psychosocial health of sexual minority persons, and (8) Psychologists recognize the positive aspects of being a sexual minority person and the individual and collective ways that sexual minority persons display resilience and resistance to stigma and oppression. 


\section{$\underline{\text { Relationships and Family }}$}

This section includes guidelines around understanding romantic and familial relationships for sexual minority persons and includes the following: (9) Psychologists strive to be knowledgeable about and respect diverse relationships among sexual minority persons, (10) Psychologists recognize the importance and complexity of sexual health in the lives of sexual minority persons, (11) Psychologists strive to understand sexual minority persons' relationships with their families of origin, as well as their families of choice, (12) Psychologists strive to understand the experiences, challenges, and strengths faced by sexual minority parents and their children.

\section{Education and Vocational Issues}

This area includes understanding how educational and workplace environments may be experienced differently by sexual minority persons and includes the following: (13) Psychologists strive to understand the educational and school system experiences that impact sexual minority students in K-12 and college/university settings, (14) Psychologists strive to understand career development and workplace issues for sexual minority persons.

\section{Professional Education, Training, and Research}

Finally, the fifth domain includes guidelines on working with trainees to educate them about sexual minority persons and conducting research that is affirmative and thoughtful about the impact of the work on the LGBTQ+ community and includes the final two guidelines: (15) Psychologists strive to educate themselves and others on psychological issues relevant to sexual minority persons, and to utilize that knowledge to improve training programs and educational systems, and (16) Psychologists strive to take an affirmative stance toward sexual minority persons and communities in all aspects of planning, conduct, dissemination, and application of research to reduce health disparities and promote psychological health and wellbeing.

\section{Examine biases before and during treatment}

Examining one's own biases is good practice for any clinician, especially when working with individuals from different backgrounds than one's own (which is almost always the case with respect to some important component of identity). According to Sue's multidimensional model of cultural competence, one must first have awareness, which includes self-awareness (Sue, 2001). Therapists are human and subject to the same biases as anyone else in society. It is important that therapists examine their own explicit or implicit biases about sexual orientation and gender in the context of working with sexual minority clients. Clinical supervision and consultation may be effective spaces to explore and challenge biases to ensure provision of ethical and high-quality care. Sexual minority communities are diverse with respect to specific sexual orientation identities, as well as all other facets of identity. Further, all people, including those who identify as sexual minorities, have been exposed to heteronormative messaging because of living in a heterosexist society. Therapists who also identify as sexual minority 
persons, in particular, may have had their own experiences navigating sexual orientation-related stigma and discrimination, which could impact one's clinical work (Pepping et al., 2018). As such, all therapists, including those who are members of the LGBTQ+ community, may benefit from such exploration of biases.

\section{Understand the Issues}

Part of being able to provide affirming care is understanding the nature and context of the situations that occur for sexual minority clients (American Psychological Association, 2012). Although it is important for clients to provide their own narratives of their experiences as a sexual minority person, it is also important for therapists to seek additional resources to learn about the community, specific health issues, relationship considerations, and other factors that may affect treatment. As is true when working with all historically excluded populations, it is incumbent upon the therapist, as the professional in the position of power, to seek their own knowledge and not rely exclusively on the client to explain customs. Over-reliance on the client for information beyond their own specific experiences (e.g., education about the LGBTQ+ community) can unfairly burden the client and may cause a rupture in the therapeutic relationship. Thus, it is important that the clinician seek their own information and resources, while simultaneously grounding this broad knowledge in the clients' unique experiences to avoid generalizations.

\section{Consider the Context}

The sociocultural environment and contextual factors that impact sexual minority clients' lives should always be considered in delivering evidence-based treatments (American Psychological Association, 2012). For example, exposure hierarchies are commonly used in treating anxiety disorders. However, an inherent assumption of these hierarchies is that the situations that are being selected are not, in fact, dangerous, but are perceived to be such. For example, for someone with social anxiety disorder, this could be a fear of ordering a coffee for fear of being judged by how the order is placed or fearing that others may stare at them. Typically, the client would be encouraged to do a "behavioral experiment" when they would go to a coffee shop and order and the client would learn this is not a situation to fear. For a sexual minority client, it is possible that even this example could expose them to experiences of discrimination compromising their physical safety, depending on their context. Recognizing real versus perceived threat and helping the client to assess whether this is a situation they are willing to expose themselves to is more helpful then sending the individual into a situation that could potentially be harmful or expose them to an anti-LGBTQ+ violence, which should always be avoided.

Finally, for those clients who require residential treatment, such as those with severe substance use disorders, patient safety should always precede other treatment considerations. Residential treatment programs should be assessed to ensure they are affirming of sexual minority clients prior to recommending these treatment programs. Additionally, because Narcotics Anonymous (NA) and Alcoholics Anonymous (AA) have a faith-based origin and focus on a "higher power," this can be unfavorable for some sexual minority clients, particularly those who have had 
negative personal experiences with religious clergy or communities. When possible, LGBTQ+ specific NA and AA groups or other sources of group support are recommended.

\section{Assessment and Context}

The focus of this chapter is on the state of psychosocial interventions for sexual minority populations. Related to appreciating the role context plays in intervention, understanding the social and structural context is also important for assessment and measurement purposes. For instance, emerging research suggests that clinical providers are more inclined to provide diagnoses of more severe forms of psychopathology (e.g., borderline personality disorder) among sexual minorities than heterosexual persons (Eubanks-Carter and Goldfried, 2006; Rodriguez-Seijas, Morgan and Zimmerman, 2021b). Relatedly, research using populationbased data further shows that sexual minority persons are more likely to endorse several criteria of severe psychiatric disorders if one does not account for associated distress or impairment related to those criteria. When one accounts for whether the behaviors are associated with distress or impairment related to borderline personality disorder symptoms, for example, the endorsement disparity is essentially halved (Rodriguez-Seijas, Morgan and Zimmerman, 2021a). The net effect might serve to stigmatize an already marginalized community through a viewing culturally normative behavior as pathology, predisposing sexual minority persons to stigma from healthcare environments related to more severe psychiatric diagnoses, and potentially routing sexual minority persons to treatments that, while intensive, might lack an LGBTQ+ affirming stance.

\section{Cultural Humility}

Cultural humility contrasts with the notion of "cultural competence" by underscoring the reality that no one therapist can ever know all there is to know about any one cultural group, even if the therapist identifies as a member of that same group. When working with sexual minority clients (and all clients), clinicians must be willing to be wrong, ask questions, and practice cultural humility. Cultural humility is a component of holding a multicultural orientation and is associated with repairing therapeutic ruptures following a microaggression (Davis et al., 2016) and better therapy outcomes (Owen et al., 2016). It is important that therapists working with this population are willing to recognize their own biases and knowledge deficits. Part of providing affirming care is being able to honestly acknowledge gaps in knowledge and mistakes in session with clients to avoid and/or repair ruptures in alliance.

\subsection{Conclusion}

In conclusion, there are a variety of evidence-based mental health treatments that have already been adapted for use with sexual minority clients. We have provided an overview of existing research, recommendations on how to adapt existing interventions, and clinical approaches to working with sexual minority clients. Although a comprehensive review was beyond the scope of this chapter, we refer readers to the Handbook of Evidence-Based Mental Health Practice with Sexual and Gender Minorities (Pachankis and Safren, 2019) as an additional resource. 
Based on our review, interventions that have been tested rigorously in randomized controlled trials have primarily focused on sexual minority men. There is still much work to be done in conducting similarly rigorous research for interventions for sexual minority women. Although not specifically reviewed here, more research is also needed on the application of evidence-based treatments to transgender and gender diverse clients, who are also underrepresented in the current evidence-base. Several of the principles described within this chapter may be applicable to gender diverse clients as well, although rigorous testing of interventions tailored to gender diverse clients are still in their infancy. Further, although the focus of our chapter was on addressing psychological disorders and stress-sensitive conditions associated with minority stress experiences, there is room for the inclusion of positive psychology principles in treatment as well. Shifting to a strengths-based approach that focuses on improving clients' lives by strengthening their understanding of self, relationships, and connection to meaningful pursuits and can also address underlying mechanisms of minority stress by capitalizing on intrinsic resilience (Lytle et al., 2014). The number of individuals identifying within the sexual and gender minority umbrella in the U.S. and the world are steadily increasing and the numbers of therapy clients identifying as such is also likely to increase (Newport, 2018). As such, we emphasize that it is imperative that clinicians have some basic competencies in working with LGBTQ+ clients and hope this chapter can serve as a practical resource in adapting existing interventions. 


\subsection{References}

American Psychological Association (2012) 'Guidelines for Psychological Practice with Lesbian, Gay, and Bisexual Clients', American Psychologist, 67(1), pp. 10-42.

APA Task Force on Psychological Practice with Sexual Minority Persons (2021) APA Guidelines for Psychological Practice with Sexual Minority Persons. American Psychological Association. Available at: www.apa.org/about/policy/psychological-practice-sexual-minority-persons.pdf.

Austin, S. B. et al. (2012) 'Eating Disorder Symptoms and Obesity at the Intersections of Gender, Ethnicity, and Sexual Orientation in US High School Students', American Journal of Public Health, 103(2), pp. e16-e22. doi: 10.2105/AJPH.2012.301150.

Bailey, J. M. et al. (2016) 'Sexual Orientation, Controversy, and Science', Psychological Science in the Public Interest, 17(2), pp. 45-101. doi: 10.1177/1529100616637616.

Barlow, D. H. et al. (2017) 'The Unified Protocol for Transdiagnostic Treatment of Emotional Disorders Compared With Diagnosis-Specific Protocols for Anxiety Disorders: A Randomized Clinical Trial', JAMA psychiatry, 74(9), pp. 875-884. doi: 10.1001/jamapsychiatry.2017.2164.

Barlow, D. H., Allen, L. B. and Choate, M. L. (2004) 'Toward a unified treatment for emotional disorders', Behavior Therapy, 35(2), pp. 205-230. doi: 10.1016/S0005-7894(04)80036-4.

Bjornsson, A. S., Didie, E. R. and Phillips, K. A. (2010) 'Body dysmorphic disorder', Dialogues in Clinical Neuroscience, 12(2), pp. 221-232.

Blashill, A. J. (2010) 'Elements of male body image: Prediction of depression, eating pathology and social sensitivity among gay men', Body Image, 7(4), pp. 310-316. doi:

10.1016/j.bodyim.2010.07.006.

Blashill, A. J. et al. (2017) Cognitive behavioral therapy for body image and self-care (CBT$B I S C)$ in sexual minority men living with HIV: A randomized controlled trial. - PsycNET. Available at: /doiLanding?doi=10.1037\%2Fhea0000505 (Accessed: 16 October 2020).

Blashill, A. J., Brown, T. A. and Klimek, P. (2019) 'Evidence-Based Practice for the Prevention and Treatment of Body Image Disturbance and Eating Pathology in Sexual Minority Men', in Pachankis, J. E. and Safren, S. A. (eds) Handbook of Evidence-Based Mental Health Practice with Sexual and Gender Minorities. New York, NY: Oxford University Press, pp. 291-312.

Bränström, R. and Pachankis, J. E. (2018) 'Sexual orientation disparities in the co-occurrence of substance use and psychological distress: a national population-based study (2008-2015)', Social Psychiatry and Psychiatric Epidemiology, 53(4), pp. 403-412. doi: 10.1007/s00127-0181491-4.

Breslau, N. (2009) 'The epidemiology of trauma, PTSD, and other posttrauma disorders', Trauma, Violence \& Abuse, 10(3), pp. 198-210. doi: 10.1177/1524838009334448.

Brim, O. G. et al. (1999) 'Midlife in the United States (MIDUS 1), 1995-1996: Version 19'. InterUniversity Consortium for Political and Social Research. doi: 10.3886/ICPSR02760.V19. 
Brown, T. A. et al. (2017) 'A randomized controlled trial of The Body Project: More Than Muscles for men with body dissatisfaction', International Journal of Eating Disorders, 50(8), pp. 873-883. doi: 10.1002/eat.22724.

Brown, T. A. and Keel, P. K. (2015) 'A randomized controlled trial of a peer co-led dissonancebased eating disorder prevention program for gay men', Behaviour Research and Therapy, 74, pp. 1-10. doi: 10.1016/j.brat.2015.08.008.

Calzo, J. P. et al. (2017) 'Eating Disorders and Disordered Weight and Shape Control Behaviors in Sexual Minority Populations', Current Psychiatry Reports, 19(8), p. 49. doi: 10.1007/s11920017-0801-y.

Carlat, D. J., Camargo, C. A. and Herzog, D. B. (1997) 'Eating disorders in males: a report on 135 patients', American Journal of Psychiatry, 154(8), pp. 1127-1132. doi: 10.1176/ajp.154.8.1127.

Carrico, A. W. et al. (2013) 'Positive affect and processes of recovery among treatment-seeking methamphetamine users', Drug and Alcohol Dependence, 132(3), pp. 624-629. doi:

10.1016/j.drugalcdep.2013.04.018.

Carrico, A. W., Gómez, W., et al. (2015) 'Pilot randomized controlled trial of an integrative intervention with methamphetamine-using men who have sex with men', Archives of Sexual Behavior, 44(7), pp. 1861-1867. doi: 10.1007/s10508-015-0505-5.

Carrico, A. W., Nation, A., et al. (2015) 'Pilot trial of an expressive writing intervention with HIVpositive methamphetamine-using men who have sex with men.', 29, 2, pp. 277-282.

Carrico, A. W. et al. (2016) 'A community-engaged randomized controlled trial of an integrative intervention with HIV-positive, methamphetamine-using men who have sex with men', BMC public health, 16, p. 673. doi: 10.1186/s12889-016-3325-1.

Carrico, A. W. et al. (2018) 'Randomized controlled trial of a positive affect intervention for methamphetamine users', Drug and Alcohol Dependence, 192, pp. 8-15. doi:

10.1016/j.drugalcdep.2018.07.029.

Carrico, A. W. et al. (2019) 'Randomized controlled trial of a positive affect intervention to reduce HIV viral load among sexual minority men who use methamphetamine', Journal of the International AIDS Society, 22(12), p. e25436. doi: 10.1002/jia2.25436.

Cochran, S. D., Björkenstam, C. and Mays, V. M. (2017) 'Sexual orientation differences in functional limitations, disability, and mental health services use: Results from the 2013-2014 National Health Interview Survey.', Journal of Consulting and Clinical Psychology, 85(12), p. 1111. doi: $10.1037 / \mathrm{ccp} 0000243$.

Cochran, S. D., Grella, C. E. and Mays, V. M. (2012) 'Do substance use norms and perceived drug availability mediate sexual orientation differences in patterns of substance use? Results from the California Quality of Life Survey II', Journal of Studies on Alcohol and Drugs, 73(4), pp. 675-685. doi: 10.15288/jsad.2012.73.675.

Cochran, S. D., Mays, V. M. and Sullivan, J. G. (2003) 'Prevalence of mental disorders, psychological distress, and mental health services use among lesbian, gay, and bisexual adults 
in the United States', Journal of Consulting and Clinical Psychology, 71(1), pp. 53-61. doi: 10.1037//0022-006x.71.1.53.

Craig, S. L. et al. (2019) 'An Affirmative Coping Skills Intervention to Improve the Mental and Sexual Health of Sexual and Gender Minority Youth (Project Youth AFFIRM): Protocol for an Implementation Study', JMIR Research Protocols, 8(6), p. e13462. doi: 10.2196/13462.

Craig, S. L. and Austin, A. (2016) 'The AFFIRM open pilot feasibility study: A brief affirmative cognitive behavioral coping skills group intervention for sexual and gender minority youth', Children and Youth Services Review, 64, pp. 136-144. doi: 10.1016/j.childyouth.2016.02.022.

Craig, S. L., Austin, A. and Alessi, E. (2013) 'Gay affirmative cognitive behavioral therapy for sexual minority youth: A clinical adaptation', Clinical Social Work Journal, 41(3), pp. 258-266. doi: $10.1007 / \mathrm{s} 10615-012-0427-9$.

Davis, D. E. et al. (2016) 'Microaggressions and Perceptions of Cultural Humility in Counseling', Journal of Counseling \& Development, 94(4), pp. 483-493. doi: 10.1002/jcad.12107.

Easton, D. et al. (2007) 'Space: The New Frontier in HIV Prevention For Young Men Who Have Sex With Men', AIDS Education and Prevention, 19(6), pp. 465-478. doi:

10.1521/aeap.2007.19.6.465.

Eaton, N. R. et al. (2015) 'Transdiagnostic factors of psychopathology and substance use disorders: a review', Social Psychiatry and Psychiatric Epidemiology, 50(2), pp. 171-182. doi: 10.1007/s00127-014-1001-2.

Eubanks-Carter, C. and Goldfried, M. R. (2006) 'The impact of client sexual orientation and gender on clinical judgments and diagnosis of borderline personality disorder', Journal of Clinical Psychology, 62(6). doi: 10.1002/jclp.20265.

Fairburn, C. G., Cooper, Z. and Shafran, R. (2003) 'Cognitive behaviour therapy for eating disorders: a "transdiagnostic" theory and treatment', Behaviour Research and Therapy, 41 (5), pp. 509-528. doi: 10.1016/S0005-7967(02)00088-8.

Farchione, T. J. et al. (2012) 'Unified protocol for transdiagnostic treatment of emotional disorders: a randomized controlled trial', Behavior Therapy, 43(3), pp. 666-678. doi: 10.1016/j.beth.2012.01.001.

Farmer, L. B., Welfare, L. E. and Burge, P. L. (2013) 'Counselor Competence With Lesbian, Gay, and Bisexual Clients: Differences Among Practice Settings', Journal of Multicultural Counseling and Development, 41(4), pp. 194-209. doi: 10.1002/j.2161-1912.2013.00036.x.

Feldman, M. B. and Meyer, I. H. (2007) 'Eating disorders in diverse lesbian, gay, and bisexual populations', The International Journal of Eating Disorders, 40(3), pp. 218-226. doi: 10.1002/eat.20360.

Foa, E. B. (2011) 'Prolonged exposure therapy: past, present, and future', Depression and Anxiety, 28(12), pp. 1043-1047. doi: 10.1002/da.20907.

Foa, E. B., Hembree, E. A. and Rothbaum, B. O. (2007) Prolonged exposure therapy for PTSD: Emotional processing of traumatic experiences: Therapist guide. New York, NY, US: Oxford 
University Press (Prolonged exposure therapy for PTSD: Emotional processing of traumatic experiences: Therapist guide), pp. viii, 146. doi: 10.1093/med:psych/9780195308501.001.0001.

Friedman, M. S. et al. (2011) 'A Meta-Analysis of Disparities in Childhood Sexual Abuse, Parental Physical Abuse, and Peer Victimization Among Sexual Minority and Sexual Nonminority Individuals', American Journal of Public Health, 101(8), pp. 1481-1494. doi: 10.2105/AJPH.2009.190009.

Garcia, J. et al. (2015) “You're Really Gonna Kick Us All Out?” Sustaining Safe Spaces for Community-Based HIV Prevention and Control among Black Men Who Have Sex with Men', PLOS ONE. Edited by H. Shang, 10(10), p. e0141326. doi: 10.1371/journal.pone.0141326.

Gómez, W. et al. (2018) 'Optimizing Contingency Management With Methamphetamine-Using Men Who Have Sex With Men', Cognitive and Behavioral Practice, 25(2), pp. 286-295. doi: 10.1016/j.cbpra.2017.08.003.

Graham, S. R., Carney, J. S. and Kluck, A. S. (2012) 'Perceived Competency in Working With LGB Clients: Where Are We Now?', Counselor Education and Supervision, 51(1), pp. 2-16. doi: 10.1002/j.1556-6978.2012.00001.x.

Halkitis, P. N., Parsons, J. T. and Stirratt, M. J. (2001) 'A double epidemic: crystal methamphetamine drug use in relation to HIV transmission among gay men', Journal of Homosexuality, 41(2), pp. 17-35. doi: 10.1300/J082v41n02_02.

Jernigan, M. M. et al. (2015) \#racialtraumaisreal. Chestnut Hill, MA: Institute for the Study and Promotion of Race and Culture at Boston College. Available at: www.bc.edu/content/dam/files/schools/Lynch School_sites/isprc/pdf/racialtraumaisrealManuscript.pdf (Accessed: 26 October 2020).

Juyal, D. et al. (2017) 'Sexual abuse in males: An underreported issue', Indian journal of sexually transmitted diseases and AIDS, 38(2), pp. 187-188. doi: 10.4103/ijstd.IJSTD_80_16.

Kaysen, D., Lehavot, K. and Dworkin, E. R. (2019) 'Application of Evidence-Based Practices for Trauma-Related Disorders Among Sexual Minority Women', in Pachankis, J. E. and Safren, S. A. (eds) Handbook of Evidence-Based Mental Health Practice with Sexual and Gender Minorities. New York, NY: Oxford University Press, pp. 244-267.

Kotov, R. et al. (2017) 'The Hierarchical Taxonomy of Psychopathology (HiTOP): A dimensional alternative to traditional nosologies', Journal of Abnormal Psychology, 126(4), pp. 454-477. doi: 10.1037/abn0000258.

Layland, E. K. et al. (2020) 'A systematic review of stigma in sexual and gender minority health interventions', Translational Behavioral Medicine, 10(5), pp. 1200-1210. doi: 10.1093/tbm/ibz200.

Le Grange, D. and Lock, J. (2007) Treating Bulimia in Adolescents: A Family-Based Approach. New York: Guilford.

Lock, J. and Le Grange, D. (2013) Treatment Manual for Anorexia Nervosa: A Family-Based Approach. 2nd. New York: Guilford. 
Lytle, M. C. et al. (2014) 'Working with LGBT Individuals: Incorporating Positive Psychology into Training and Practice', Psychology of Sexual Orientation and Gender Diversity, 1(4), pp. 335347. doi: $10.1037 / \mathrm{sgd} 0000064$.

Matthews-Ewald, M. R., Zullig, K. J. and Ward, R. M. (2014) 'Sexual orientation and disordered eating behaviors among self-identified male and female college students', Eating Behaviors, 15(3), pp. 441-444. doi: 10.1016/j.eatbeh.2014.05.002.

Mayer, K. H., Colfax, G. and Guzman, R. (2006) 'Club Drugs and HIV Infection: A Review', Clinical Infectious Diseases, 42(10), pp. 1463-1469. doi: 10.1086/503259.

Meneguzzo, P. et al. (2018) 'Eating disorders symptoms in sexual minority women: A systematic review', European Eating Disorders Review, 26(4), pp. 275-292. doi: 10.1002/erv.2601.

Menza, T. W. et al. (2010) 'Contingency management to reduce methamphetamine use and sexual risk among men who have sex with men: a randomized controlled trial', BMC public health, 10, p. 774. doi: 10.1186/1471-2458-10-774.

Meyer, I. H. (2003) 'Prejudice, Social Stress, and Mental Health in Lesbian, Gay, and Bisexual Populations: Conceptual Issues and Research Evidence', Psychological bulletin, 129(5), pp. 674-697. doi: 10.1037/0033-2909.129.5.674.

Miller, W. R. and Rollnick, S. (2012) Motivational Interviewing: Helping People Change. Guilford Press.

Mimiaga, M. J. et al. (2012) 'A pilot trial of integrated behavioral activation and sexual risk reduction counseling for HIV-uninfected men who have sex with men abusing crystal methamphetamine', AIDS patient care and STDs, 26(11), pp. 681-693. doi: 10.1089/apc.2012.0216.

Mimiaga, M. J. et al. (2018) 'A randomized controlled efficacy trial of behavioral activation for concurrent stimulant use and sexual risk for HIV acquisition among MSM: project IMPACT study protocol', BMC public health, 18(1), p. 914. doi: 10.1186/s12889-018-5856-0.

Mimiaga, M. J. et al. (2019) 'An initial randomized controlled trial of behavioral activation for treatment of concurrent crystal methamphetamine dependence and sexual risk for HIV acquisition among men who have sex with men', AIDS care, 31(9), pp. 1083-1095. doi: 10.1080/09540121.2019.1595518.

Newport, F. (2018) In U.S., Estimate of LGBT Population Rises to 4.5\%. Available at: https://news.gallup.com/poll/234863/estimate-lgbt-population-rises.aspx (Accessed: 16 October 2020).

'Obsessive-Compulsive and Related Disorders' (2013) in Diagnostic and Statistical Manual of Mental Disorders Fifth Edition (DSM-5). 5th edn. American Psychiatric Association. Available at: https://doi.org/10.1176/appi.books.9780890425596.dsm06.

O'Cleirigh, C. et al. (2019) 'Cognitive Behavioral Therapy for Trauma and Self-Care (CBT-TSC) in Men Who have Sex with Men with a History of Childhood Sexual Abuse: A Randomized Controlled Trial', AIDS and behavior, 23(9), pp. 2421-2431. doi: 10.1007/s10461-019-02482-z. 
O'Cleirigh, C., Safren, S. A. and Mayer, K. H. (2012) 'The Pervasive Effects of Childhood Sexual Abuse: Challenges for Improving HIV Prevention and Treatment Interventions', Journal of acquired immune deficiency syndromes (1999), 59(4), pp. 331-334. doi:

10.1097/QAI.0b013e31824aed80.

Owen, J. et al. (2016) 'Client perceptions of therapists' multicultural orientation: Cultural (missed) opportunities and cultural humility.', Professional Psychology: Research and Practice, 47(1), pp. 30-37. doi: 10.1037/pro0000046.

Pachankis, J. E. (2007) 'The psychological implications of concealing a stigma: A cognitiveaffective-behavioral model', Psychological Bulletin, 133(2), pp. 328-345. doi: 10.1037/00332909.133.2.328.

Pachankis, J. E. (2014) 'Uncovering Clinical Principles and Techniques to Address Minority Stress, Mental Health, and Related Health Risks Among Gay and Bisexual Men', Clinical Psychology: A Publication of the Division of Clinical Psychology of the American Psychological Association, 21(4), pp. 313-330. doi: 10.1111/cpsp.12078.

Pachankis, J. E. (2015) 'A transdiagnostic minority stress treatment approach for gay and bisexual men's syndemic health conditions', Archives of Sexual Behavior, 44(7), pp. 18431860. doi: 10.1007/s10508-015-0480-x.

Pachankis, J. E. et al. (2015) 'LGB-affirmative cognitive-behavioral therapy for young adult gay and bisexual men: A randomized controlled trial of a transdiagnostic minority stress approach.', Journal of Consulting and Clinical Psychology, 83(5), pp. 875-889. doi: 10.1037/ccp0000037.

Pachankis, J. E. (2018) 'The scientific pursuit of sexual and gender minority mental health treatments: Toward evidence-based affirmative practice', The American Psychologist, 73(9), pp. 1207-1219. doi: 10.1037/amp0000357.

Pachankis, J. E. et al. (2019) 'Project ESTEEM protocol: a randomized controlled trial of an LGBTQ-affirmative treatment for young adult sexual minority men's mental and sexual health', BMC Public Health, 19(1). doi: 10.1186/s12889-019-7346-4.

Pachankis, J. E. et al. (2020) 'A transdiagnostic minority stress intervention for gender diverse sexual minority women's depression, anxiety, and unhealthy alcohol use: A randomized controlled trial.', Journal of Consulting and Clinical Psychology, 88(7), pp. 613-630. doi: $10.1037 / \mathrm{ccp} 0000508$.

Pachankis, J. E. and Hatzenbuehler, M. L. (2013) 'The Social Development of Contingent SelfWorth in Sexual Minority Young Men: An Empirical Investigation of the "Best Little Boy in the World" Hypothesis', Basic and Applied Social Psychology, 35(2), pp. 176-190. doi: 10.1080/01973533.2013.764304.

Pachankis, J. E. and Safren, S. A. (eds) (2019) Handbook of Evidence-Based Mental Health Practice with Sexual and Gender Minorities. New York, NY: Oxford University Press.

Pakula, B. et al. (2016) 'Prevalence and Co-Occurrence of Heavy Drinking and Anxiety and Mood Disorders Among Gay, Lesbian, Bisexual, and Heterosexual Canadians', American Journal of Public Health, 106(6), pp. 1042-1048. doi: 10.2105/AJPH.2016.303083. 
Parsons, J. T. et al. (2014) 'A randomized controlled trial utilizing motivational interviewing to reduce HIV risk and drug use in young gay and bisexual men.', 82(1), pp. 9-18.

Rawson, R. A. et al. (2004) 'A multi-site comparison of psychosocial approaches for the treatment of methamphetamine dependence', Addiction (Abingdon, England), 99(6), pp. 708717. doi: 10.1111/j.1360-0443.2004.00707.x.

Reback, C. J. et al. (2010) 'Contingency management among homeless, out-of-treatment men who have sex with men', Journal of Substance Abuse Treatment, 39(3), pp. 255-263. doi: 10.1016/j.jsat.2010.06.007.

Resick, P. A. and Schnicke, M. K. (1993) Cognitive processing therapy for rape victims: A treatment manual. Thousand Oaks, CA, US: Sage Publications, Inc (Cognitive processing therapy for rape victims: A treatment manual), pp. xi, 178.

Rock, M., Carlson, T. S. and McGeorge, C. R. (2010) 'Does Affirmative Training Matter? Assessing CFT Students' Beliefs About Sexual Orientation and Their Level of Affirmative Training', Journal of Marital and Family Therapy, 36(2), pp. 171-184. doi: 10.1111/j.17520606.2009.00172.x.

Rodriguez-Seijas, C., Eaton, N. R. and Pachankis, J. E. (2019) 'Prevalence of psychiatric disorders at the intersection of race and sexual orientation: Results from the National Epidemiologic Survey of Alcohol and Related Conditions-III', Journal of Consulting and Clinical Psychology, 87(4), pp. 321-331. doi: 10.1037/ccp0000377.

Rodriguez-Seijas, C., Morgan, T. A. and Zimmerman, M. (2021a) 'A Population-Based Examination of Criterion-Level Disparities in the Diagnosis of Borderline Personality Disorder Among Sexual Minority Adults', Assessment, 28(4), pp. 1097-1109. doi:

$10.1177 / 1073191121991922$.

Rodriguez-Seijas, C., Morgan, T. A. and Zimmerman, M. (2021b) 'Is There a Bias in the Diagnosis of Borderline Personality Disorder Among Lesbian, Gay, and Bisexual Patients?', Assessment, 28(3), pp. 724-738. doi: 10.1177/1073191120961833.

Rogers, B. G. et al. (2019) 'I Wasn't in My Right Mind”: Qualitative Findings on the Impact of Alcohol on Condom Use in Patients Living with HIV/AIDS in Brazil, Thailand, and Zambia (HPTN 063)', International Journal of Behavioral Medicine, 26(1), pp. 17-27. doi: 10.1007/s12529-018-9739-7.

Roll, J. M. et al. (2006) 'Contingency management for the treatment of methamphetamine use disorders', The American Journal of Psychiatry, 163(11), pp. 1993-1999. doi: 10.1176/ajp.2006.163.11.1993.

Saewyc, E. M. (2011) 'RESEARCH ON ADOLESCENT SEXUAL ORIENTATION: DEVELOPMENT, HEALTH DISPARITIES, STIGMA AND RESILIENCE', Journal of Research on Adolescence: The Official Journal of the Society for Research on Adolescence, 21(1), pp. 256-272. doi: 10.1111/j.1532-7795.2010.00727.x.

Safer, D. L., Couturier, J. L. and Lock, J. (2007) 'Dialectical Behavior Therapy Modified for Adolescent Binge Eating Disorder: A Case Report', Cognitive and Behavioral Practice, 14(2), pp. 157-167. doi: 10.1016/j.cbpra.2006.06.001. 
Safer, D. L., Telch, C. F. and Agras, W. S. (2001) 'Dialectical behavior therapy for bulimia nervosa', The American Journal of Psychiatry, 158(4), pp. 632-634. doi:

10.1176/appi.ajp.158.4.632.

Sanders, J. M. (2020) 'Seeking Acceptance: LGBTQ and Membership in Alcoholics Anonymous (AA)', Alcoholism Treatment Quarterly, 0(0), pp. 1-16. doi: 10.1080/07347324.2020.1738295.

Sauer-Zavala, S. et al. (2020) 'Does the unified protocol really change neuroticism? Results from a randomized trial', Psychological Medicine, pp. 1-10. doi: 10.1017/S0033291720000975.

Shoptaw, S. et al. (2005) 'Behavioral treatment approaches for methamphetamine dependence and HIV-related sexual risk behaviors among urban gay and bisexual men', Drug and Alcohol Dependence, 78(2), pp. 125-134. doi: 10.1016/j.drugalcdep.2004.10.004.

Shoptaw, S. et al. (2008) 'Outcomes using two tailored behavioral treatments for substance abuse in urban gay and bisexual men', Journal of Substance Abuse Treatment, 35(3), pp. 285293. doi: 10.1016/j.jsat.2007.11.004.

Simari, C. G. and Baskin, D. (1982) 'Incestuous experiences within homosexual populations: A preliminary study', Archives of Sexual Behavior, 11(4), pp. 329-344. doi: 10.1007/BF01541594.

Starks, T. J. et al. (2018) 'Motivational interviewing with couples: A theoretical framework for clinical practice illustrated in substance use and HIV prevention intervention with gay male couples', Psychology of Sexual Orientation and Gender Diversity, 5(4), pp. 490-502. doi: $10.1037 /$ sgd0000297.

Starks, T. J. et al. (2020) 'Motivational interviewing with male couples to reduce substance use and HIV risk: Manifestations of partner discord and strategies for facilitating dyadic functioning', Psychotherapy, 57(1), pp. 58-67. doi: 10.1037/pst0000278.

Stemple, L. and Meyer, I. H. (2014) 'The sexual victimization of men in America: new data challenge old assumptions', American Journal of Public Health, 104(6), pp. e19-26. doi: 10.2105/AJPH.2014.301946.

Stice, E. et al. (2012) 'A Preliminary Trial of a Prototype Internet Dissonance-Based Eating Disorder Prevention Program for Young Women with Body Image Concerns', Journal of consulting and clinical psychology, 80(5), pp. 907-916. doi: 10.1037/a0028016.

Stice, E. et al. (2015) 'Effectiveness Trial of a Selective Dissonance-based Eating Disorder Prevention Program with Female College Students: Effects at 2- and 3-Year Follow-up', Behaviour research and therapy, 71, pp. 20-26. doi: 10.1016/j.brat.2015.05.012.

Stice, E., Yokum, S. and Waters, A. (2015) 'Dissonance-Based Eating Disorder Prevention Program Reduces Reward Region Response to Thin Models; How Actions Shape Valuation', PLOS ONE, 10(12). doi: 10.1371/journal.pone.0144530.

Substance Abuse and Mental Health Services Administration and U.S. Department of Health and Human Services (2018) '2018 National Survey on Drug Use and Health: Lesbian, Gay, \& Bisexual (LGB) Adults', p. 56. 
Sue, D. W. (2001) 'Multidimensional Facets of Cultural Competence', The Counseling Psychologist, 29(6), pp. 790-821. doi: 10.1177/0011000001296002.

Taylor, S. W. et al. (2018) 'Evaluating a Novel Intervention to Reduce Trauma Symptoms and Sexual Risk Taking: Qualitative Exit Interviews with Sexual Minority Men with Childhood Sexual Abuse', Psychology, health \& medicine, 23(4), pp. 454-464. doi:

10.1080/13548506.2017.1348609.

Tran, A. et al. (2020) “It's all outward appearance-based attractions": A qualitative study of body image among a sample of young gay and bisexual men', Journal of Gay \& Lesbian Mental Health, 24(3), pp. 281-307. doi: 10.1080/19359705.2019.1706683.

Watson, R. J. et al. (2017) 'Trends and disparities in disordered eating among heterosexual and sexual minority adolescents', International Journal of Eating Disorders, 50(1), pp. 22-31. doi: 10.1002/eat.22576.

Wilfley, D. E. et al. (2002) 'A randomized comparison of group cognitive-behavioral therapy and group interpersonal psychotherapy for the treatment of overweight individuals with binge-eating disorder', Archives of General Psychiatry, 59(8), pp. 713-721. doi: 10.1001/archpsyc.59.8.713.

Wilhelm, S. et al. (2014) 'Modular cognitive-behavioral therapy for body dysmorphic disorder: a randomized controlled trial', Behavior Therapy, 45(3), pp. 314-327. doi:

10.1016/j.beth.2013.12.007. 